\title{
la prevención de accidentes
}

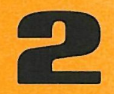

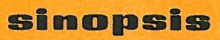

Todas las personas prefieren desempeñar sus tareas en aquellas organizaciones que tienen hacia ellos las consideraciones correspondientes a un ser humano. Uno de los medios más eficaces a disposición de cualquier empresa para elevar la moral del individuo y para crear que redundará en el doble fruto: evitar las pérdidas directas e indirectas causadas por los accidentes de trabajo que se detallaron en un trabajo anterior, y conseguir aumentar el rendimiento del operario al hacerle un ambiente más grato por la preocupación que se demues tra hacia su persona.

Es casi imposible detallar todos aquellos puntos que se deben tener en cuenta en un programa de seguridad; sin embargo, y como exponente de su diversidad, se señalan en este capítulo algunas causas, que, sin estar ligadas directamente con el trabajo individual, no obstante se encuentran íntimamente relacionadas con el tema, como son: la curiosidad pública, los desplazamientos, la higiene y el orden.

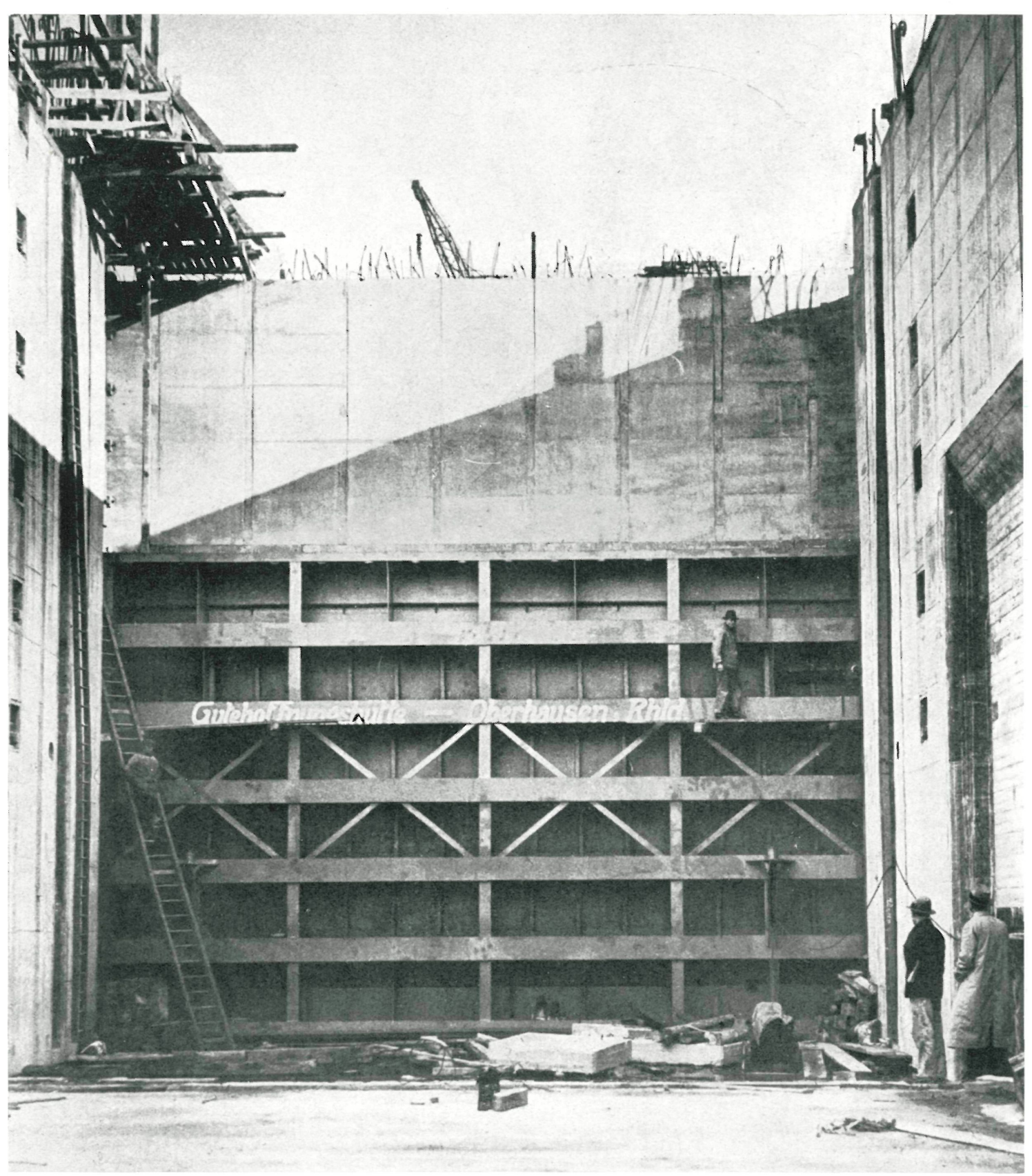


Como se expuso en el Capítulo 1, núm. 177 de esta Revista, todas las personas prefieren desempeñar sus tareas en aquellas organizaciones y lugares en los que se les hace objeto de las deferencias y consideraciones correspondientes a un ser humano, independientemente de que las necesidades del trabajo requieran integrarlos en forma más o menos impersonal dentro de un complejo cualquiera.

Uno de los medios más eficaces para conseguir esto, a disposición de una empresa cualquiera, es el de proporcionar lugares de trabajo que tengan la menor exposición y el menor riesgo posible, y mostrarle estas características al operario, indicándoles también, por medio de una eficaz y permanente prevención de accidentes, que esto es así y que existe una preocupación constante por su integridad física, a la vez que llevar a su ánimo que la organización no quiere explotarle, aun a costa de su riesgo personal, ya que con una cooperación mutua entre la empresa y él se pueden hacer desaparecer los accidentes en su casi absoluta totalidad.

De esta campaña de prevención de accidentes se obtiene, por lo tanto, un doble fruto desde el punto de vista material y de rendimiento: se logra elevar la moral del trabajador, con lo que su productividad aumenta y, además, se evitan las pérdidas directas e indirectas que motiva un accidente y que se expusieron en el ya citado Capítulo 1.

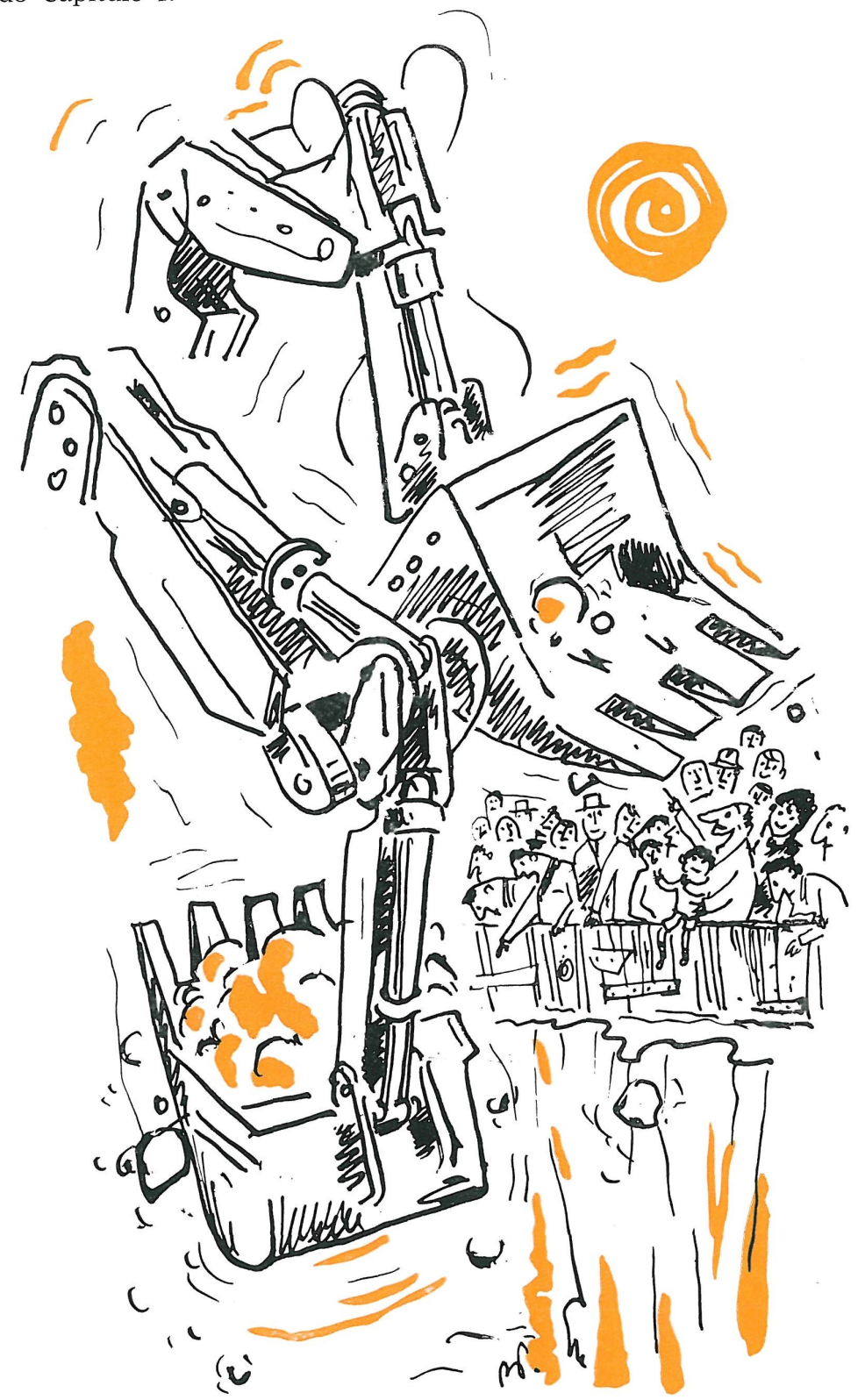

Es casi imposible poder detallar todos aquellos motivos que, o bien pueden provocar accidentes, o bien, por estar más o menos concatenados con los mismos, pueden originar consecuencias perjudiciales para el operario, para el trabajo-y, como es lógico, para la empresa que lo efectúa-y para personas ajenas al mismo; sin embargo, y como un exponente de la diversidad y variación de las circunstancias y causas que pueden estar íntimamente relacionadas con este tema, van a señalarse algunas de ellas, así como diferentes consideraciones relacionadas con las mismas, en este trabajo, dejando, para capítulos posteriores, el estudio de aquellas operaciones que con mayor frecuencia pueden motivar accidentes, por otra parte perfectamente evitables.

\section{el püblico}

Como colectividad, el público tiene una gran curiosidad por todos aquellos trabajos de obras y construcción en los que intervenga maquinaria, y mucho más si ésta es potente y complicada. Los individuos de esta colectividad son capaces, con tal de poder observar mejor los trabajos que se están efectuando, de desplazamientos incómodos y de colocarse en posiciones difíciles y peligrosas, lo que, unido a su total ignorancia, los convierten en posibles víctimas de un accidente desgraciado.

La colocación de medios de protección para evitar que puedan producirse accidentes entre los observadores del público, será un trabajo siempre rentable para el contratista de la obra. Pero hay que te- 


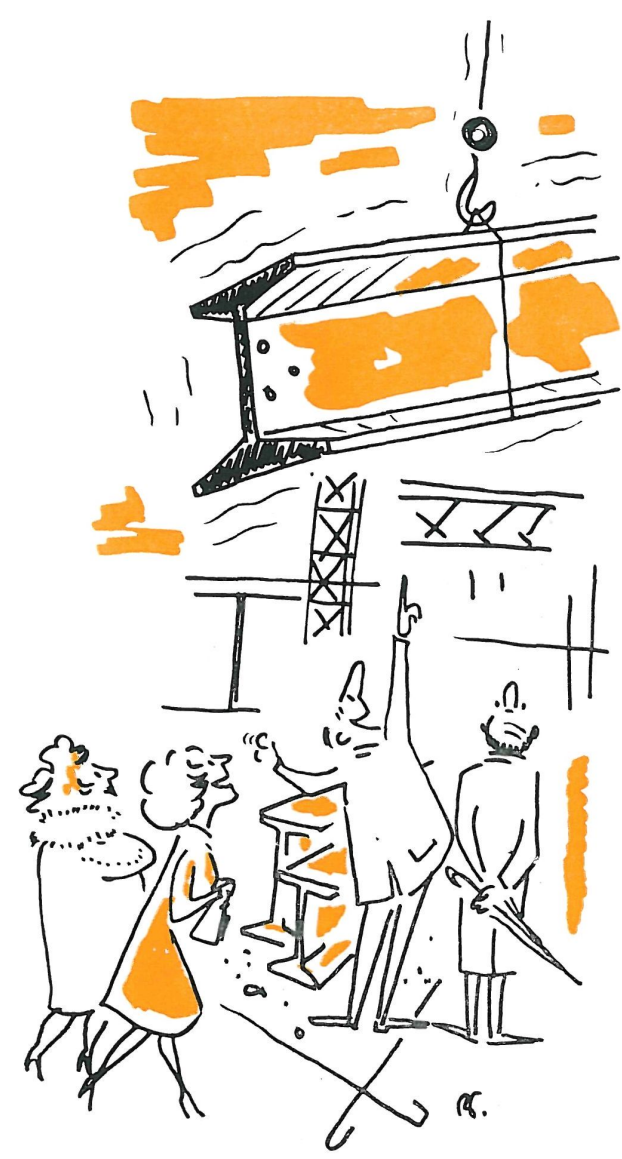

ner presente que no basta con poner medios para que no se produzca un suceso desgraciado, es preciso además que aquéllos sean los apropiados y de las características debidas; por ejemplo, el hecho de poner entre el público y una excavación una valla de $1,50 \mathrm{~m}$ de altura puede ser todavía peor que no poner nada e, incluso, revestir mayor peligrosidad aumentando la probabilidad de que ocurra una desgracia, ya que parece una invitación a que algún padre sitúe a su pequeño encima de la valla "para que vea mejor». Será preciso, por lo tanto, en este caso, una tapia de $2 \mathrm{~m}$ de altura con unas ventanillas, para poder satisfacer la curiosidad del público, y éstas, a su vez, deberán estar protegidas con tela metálica.

También el hecho de facilitar el pasaje del público por aquellos lugares en los que la ejecución de obras impida su normal desplazamiento, debe ser objeto de una cuidadosa atención por parte del constructor-algo deficiente en muchos casos-para que no sea tan sólo la evitación de un posible accidente lo que se busque, sino que a la vez se logre evitar molestias, ya que no en su totalidad, sí por lo menos en el grado suficiente para que el público se dé cuenta de que nos preocupamos por él y por proporcionarle el mínimo embarazo posible al hacerle objeto de nuestra primordial atención.

Otra de las facetas relacionadas con el público es la de la publicidad de los accidentes. Cuando ocurre un suceso desgraciado, la publicidad del mismo es siempre molesta para la organización implicada en el mismo; ya que aun en el caso más favorable de que se hagan constar en la noticia las medidas preventivas que se habían tomado para la evitación del mismo, sin embargo en el ánimo de las personas existe el estado de opinión de que nunca se han tomado las medidas estrictamente convenientes y que, por lo tanto, el accidente ha sido el fruto del descuido y de la incompetencia.

Para contrarrestar esta animadversión, más o menos encubierta, deben ser exhibidas, en las ocasiones propicias, las medidas preventivas tomadas, los programas educativos que se tengan en marcha, así como aprovechar la terminación de obras de importancia para hacer patente la carencia de accidentes ocurridos, fruto debido a la previsión y oportunidad de un programa de prevención de accidentes.

\section{Ios alesplazcamientos}

Una gran proporción de accidentes de trabajo o, por lo merıos, considerados como tales, suceden cuando los empleados se trasladan, bien de su domicilio al sitio de trabajo o viceversa, o bien a lugares a los que una misión laboral hace preciso efectuar su transporte.

Existen múltiples factores que intervienen combinadamente para acentuar la peligrosidad de estos desplazamientos:

El hecho de estar el sujeto sometido a un cambio de mentalidad es uno de ellos, ya que pasa de pensar en las ideas relacionadas con su vida de hogar, a aquellas otras ligadas a las circunstancias que le esperan en su lugar de trabajo para esa jornada, $y$, al contrario, el cese en la tarea para pensar de nuevo en su vida familiar hacen que, tanto en un sentido como en el otro, el desplazamiento lo efectúe con bastante distracción.

Por otra parte, el hecho de realizar en forma permanente un acto repetido, por lo menos diariamente, crea hábitos inevitables, en la ejecución del viaje, que al no requerir atención pueden tener, como consecuencia fatal, un accidente. El hecho de cruzar diariamente una carretera para tomar un autobús se convierte, por ejemplo, en una rutina realizada sin prestar mucha atención y que, si además es a la salida del trabajo y con miedo de perder el medio de transporte, puede efectuarse sin ningún cuidado y hasta a la carrera. 
El cambio rápido que supone pasar de una situación estable, como es la de trabajo, a la de realizar un desplazamiento, es otra de las circunstancias influyentes, puesto que requiere en el individuo una adaptación que no se realiza en forma instantánea.

El temor a llegar tarde o el efectuar los traslados a horas intempestivas, son otras de las circunstancias que concurren para que los traslados no se hagan con las debidas medidas y condiciones de seguridad.

Estos y otros factores se combinan mutuamente, influyendo también mutuamente para lograr que la proporcionalidad de accidentes-kilómetro sea muchísimo más elevada en los desplazamientos relacionados con el trabajo que en aquellos otros en los que no interviene esta circunstancia.

El piloto de una línea aérea regular decía: «La parte más peligrosa de mi misión consiste en el desplazamiento del aeropuerto a mi casa.»

No se han incluido en las consideraciones precedentes ni los transportes colectivos que pueda tener la organización, ni aquellos otros medios que puedan ser empleados para la accesibilidad del operario al lugar de trabajo; pero no quiere esto decir que no deban ser objeto también de un cuidadoso estudio, sino únicamente que por tratarse de circunstancias más directamente ligadas con la propia ocupación entran mejor en un trabajo posterior.

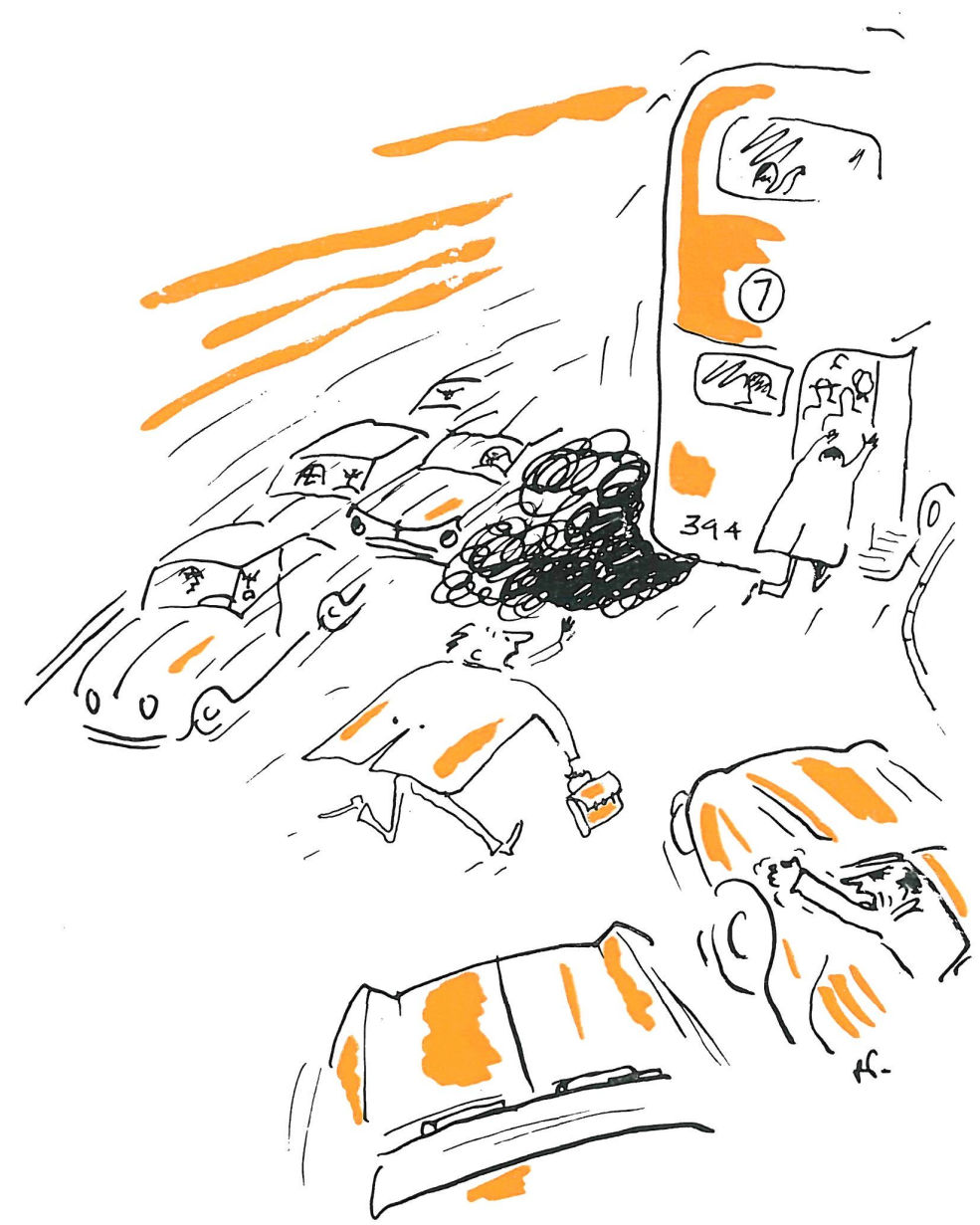

Ia salubridad thigione

Desde el grado de iluminación hasta la higiene en la alimentación y en la bebida existen múltiples factores que influyen poderosamente en el estado físico del individuo y que, a su vez, pueden ser causa directa, por falta de visibilidad, malestar, mareos, etc., de un accidente, inducido, de trabajo. Es indudable que todo lo que repercuta en el estado de salud del operario producirá un mayor o menor rendimiento del mismo.

En el caso de que no exista agua potable, por ejemplo, pero que se disponga de depósitos para extinción de incendios u otros usos, deberá hacerse constar en los tanques claramente las advertencias correspondientes al empleo indebido del agua como bebida, así como las consecuencias de su ingestión, para evitar que ante circunstancias forzadas no la ingiera nadie, evitando, de esta forma, las consecuencias correspondientes.

En los aseos deben proscribirse las toallas de uso común, siendo preferible el empleo de servicios de papel individuales. Todas otras aquellas medidas que contribuyan a elevar el bienestar físico deberán adoptarse para lograr el mayor rendimiento individual. 


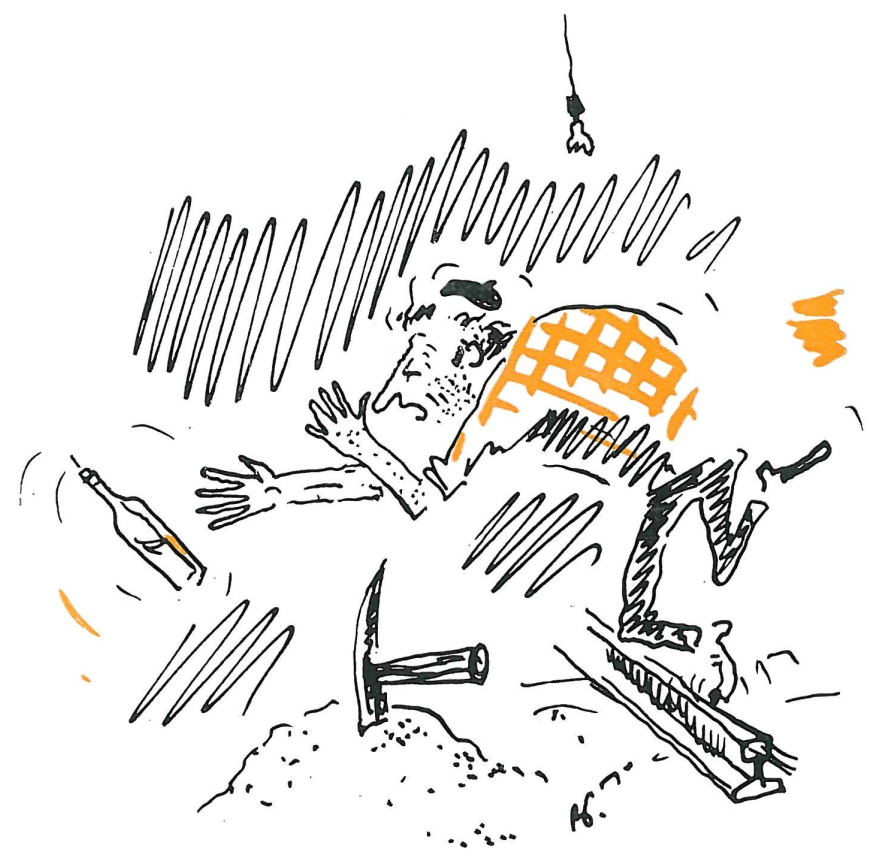

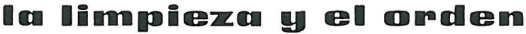

Del 30 al 35 por 100 de los accidentes de trabajo suceden por resbalamientos, tropezones o caídas. Esta proporción tan elevada muestra claramente, cómo nunca serán inútiles todas aquellas medidas que se tomen, por exageradas que parezcan, y que tiendan a lograr que en los lugares de trabajo reine la limpieza y el orden, evitando con rigurosidad que en el suelo, muros, estanterías, etc., se encuentren objetos que puedan perturbar los movimientos normales.

Esta labor no puede ser encargada a un equipo de limpieza, debe ser, en cambio, una tarea colectiva del conjunto completo y llevarse a cabo con continuidad desde la iniciación de la obra hasta su terminación, siendo considerada como el primer punto de cualquier campaña de prevención de accidentes.

No deben ser solamente las cáscaras, papeles y las tablas con clavos-tan frecuentes-, los objetos que han de perseguirse; esta labor tiene que ser completa y absoluta, la cual, además de evitar accidentes, dé una clara idea de la organización y el orden que reinan en la empresa.

\section{CHINCHILLA}

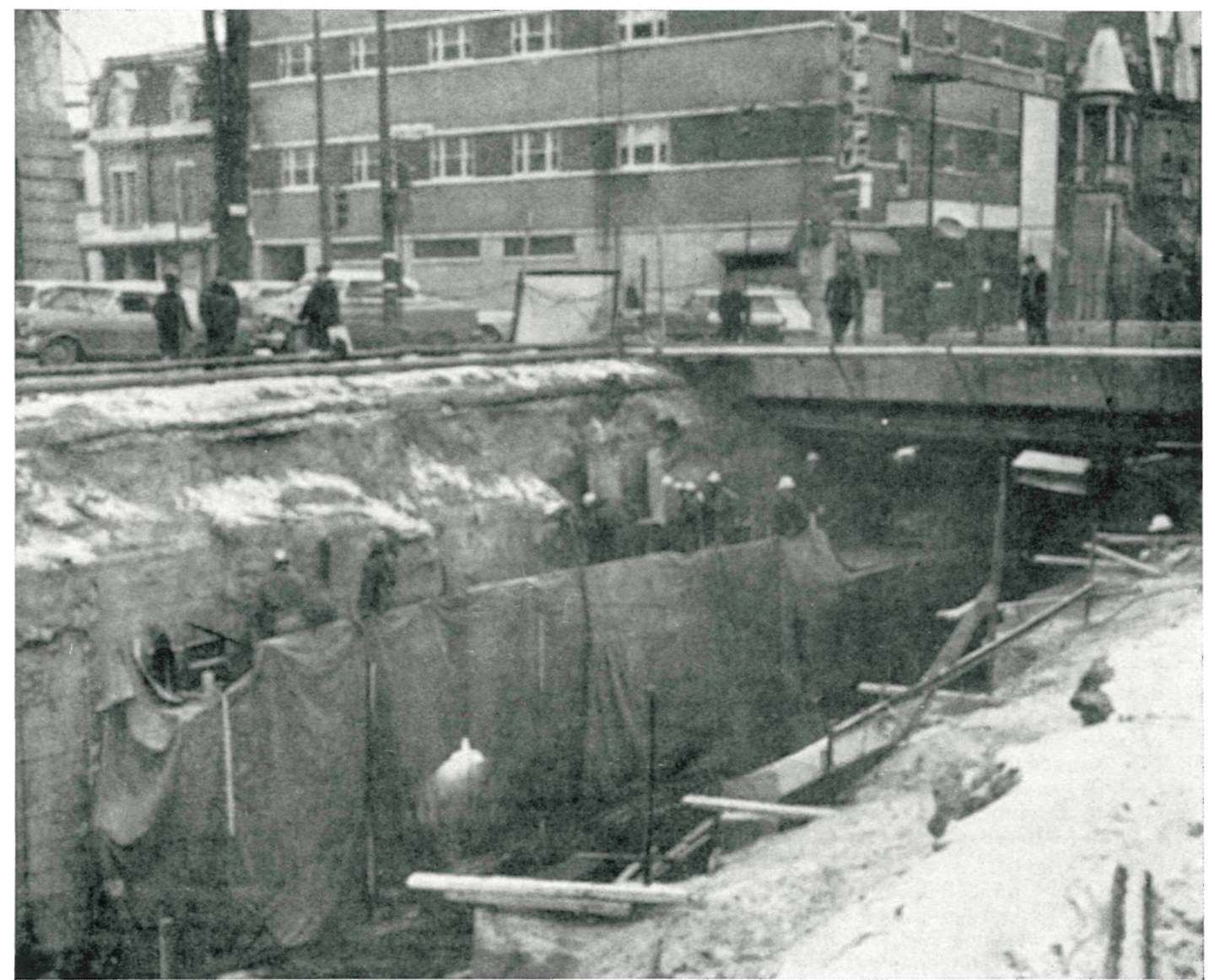




\section{La prèvention des accidemts - Chapitme IL}

M. Chinchilla.

Tout le monde, comme il est naturel, préfère travailler pour les organisations qui montrent pour leur personnel les considérations qui correspondent à un être humain. Un des moyens les plus efficaces, à portée de n'importe quelle entreprise, pour élever le moral de l'individu et créer un lien de coopération entre lui et l'entreprise est de réaliser un programme de prévention des accidents. Ce programme donnera un double fruit: éviter les pertes directes et indirectes causées par les accidents du travail, qui seront énumérées dans une autre étude, et augmenter le rendement du travailleur en lui procurant une ambiance plus agréable grâce à l'attention dont il se sentira entouré.

Il est presque impossible de détailler tous les points dont il faut tenir compte dans un programme de sécurité. Cependant et comme exemple de leur diversité, sont signalées dans ce chapitre quelques-unes des causes qui, sans être liées directement au travail individuel, se trouvent pourtant en relation étroite avec le thème dont il est question ici, tels que la curiosité pablique, les déplacement, l'hygiène et l'ordre.

\section{Accident prevention - Bhapter In}

M. Chinchilla.

Everyone prefers to work in those organisations where their dignity of human beings is respected. One of the most efficient means at the disposal of any firm, to improve the morale of the individual employees, and to establish a spirit of cooperation between the individual and the firm, is to set up a program of accident benefit. It will avoid direct and indirect losses due to working accidents, as analysed in a previous article, and it will make it possible to improve total performance of each worker, since he will work in a more favourable environment, free from worry about potential danger to his person.

It is almost impossible to describe in detail all those items that should be taken into account in a safety program. None the less, and as an indication of their diversity, some sources of danger are mentioned in this chapter, which are closely connected with human safety, such as public curiosity, travelling from one place to another, cleanliness and order.

\section{Unfallverlhütumg - Kapitel II}

M. Chinchilla.

Jedermann zieht es vor, für Organisationen zu arbeiten, die sich um das Wohlergehen Ihrer Mitarbeiter in menschlicher Rücksichtnahme bemühen. Eines der wirksamsten Mittel, das jedem Unternehmen zur Verfügung steht, um die Moral des Arbeitenden zu heben und die Zusammenarbeit zwischen demselben und dem Unternehmen zu fördern, besteht in der Verwirklichung eines Programms zur Verhütung von Unfällen. Ein solches Programm wird sich in doppelter Hinsicht vorteilhaft auswirken: Vermeidung der direkten und indirekten Verluste, verursacht durch die Arbeitsunfälle, welche in einer vorhergehenden Arbeit aufgeführt wurden, und erhöhte Leistung des Arbeiters durch eine angenehmere Gestaltung seiner Umgebung und die Aufmerksamkeit die dadurch seiner Person gegenüber zum Ausdruck kommt.

Es ist fast unmöglich, alle Gesichtspukte zu erfassen, die bei einem Sicherheitsprogramm zu berücksichtigen sind. Es werden jedoch in diesem Kapitel zur Veranschaulichung ihrer Vielfalt einige Ursachen aufgeführt, die, wenn sie auch nicht in direktem Zusammenhang mit der Arbeit des einzelnen stehen, trotzdem mit dem Thema eng verbunden sind, wie Neugier des Publikums, Versetzungen, Hygiene und Ordnung. 